\title{
On Combined Effects of Heat Transfer and Chemical Reaction for the Flow through an Asymmetric Channel with Orthogonally Deformable Porous Walls
}

\author{
Syed Tauseef Mohyud-Din, ${ }^{1}$ Naveed Ahmed, ${ }^{1}$ Umar Khan, ${ }^{1}$ Asif Waheed, \\ Saqib Hussain, ${ }^{3}$ and Maslina Darus ${ }^{4}$ \\ ${ }^{1}$ Department of Mathematics, Faculty of Sciences, HITEC University, Taxila Cantt, Pakistan \\ ${ }^{2}$ Department of Mathematics, COMSATS Institute of Information Technology, Attock, Pakistan \\ ${ }^{3}$ Department of Mathematics, COMSATS Institute of Information Technology, Abbottabad, Pakistan \\ ${ }^{4}$ School of Mathematical Sciences, Faculty of Science and Technology, Universiti Kebangsaan Malaysia, \\ 43600 Bangi, Selangor, Malaysia
}

Correspondence should be addressed to Syed Tauseef Mohyud-Din; tauseefsyedn@gmail.com

Received 19 November 2015; Revised 26 January 2016; Accepted 21 February 2016

Academic Editor: Francesco Franco

Copyright (c) 2016 Syed Tauseef Mohyud-Din et al. This is an open access article distributed under the Creative Commons Attribution License, which permits unrestricted use, distribution, and reproduction in any medium, provided the original work is properly cited.

\begin{abstract}
The combined effects of heat transfer and chemical reaction are studied for the flow through a semi-infinite asymmetric channel with orthogonally deformable porous walls. The similarity transforms have been used to reduce the conservation laws to a corresponding system of nonlinear ordinary differential equations. The resulting equations are solved, both analytically and numerically, by using Homotopy Analysis Method (HAM) and the fourth-order Runge-Kutta (RK-4) method, respectively. The convergence of the analytical solution is assured through the so-called total squared residual error analysis. The optimal values of auxiliary parameters are obtained by minimizing the total squared residual error.
\end{abstract}

\section{Introduction}

The flow between porous channels can rightly be considered as one of the most important problems in fluid mechanics. Its practical applications in diversified fields of science and technology have been the basic reason why scientists from all over the world have tried to understand these flows in a better way. These applications include uniformly distributed irrigation, interbody biological transport, ablation processes, sublimation mechanisms, propellant combustion, and industrial cleansing systems.

Formative work regarding these kinds of flows can be traced back to Berman [1], who initiated a mathematical model to describe the flows between parallel plates. He established that the normal component of the velocity must be independent of streamwise coordinate. This in fact enabled him to reduce Navier-Stokes equations to a single, fourthorder, nonlinear ordinary differential equation. Following his footsteps, numerous studies have been carried out later on.
The detailed history about these investigations can be found in [2].

Heat transfer coupled with chemical reaction plays an important role in many fields of science and engineering. Its practical applications may include combustion systems, atomic reactor safety, dying of cloths, metallurgy, and chemical engineering. A chemical reaction is said to be of first order if the rate of reaction varies directly with respect to the concentration. Almost in all chemical industries, a relatively inexpensive raw material is put through some specifically designed chemical processes to obtain high value products. In most of the situations, these chemical processes are based on chemical reactions in the presence of heat transfer. Bridges accompanied by Rajagopal [3] investigated the pulsatile flow of a chemically reacting fluid, whose viscosity was dependent on the concentration of the constituents. The flow was governed by a convection-reaction-diffusion equation and the velocity gradient, which could thicken or thin the fluid. 
The effects of chemical reaction and the space porosity on mixed convective MHD flow in an asymmetric channel were investigated by Srinivas and Muthuraj [4]. The details of heat transfer analysis can be found in [5-11].

In a very recent article, Reddy et al. [12] discussed the combined effects of heat and chemical reaction on an asymmetric laminar flow between slowly expanding and contracting walls. To make their perturbation solution valid, they had to impose physical constraints such as lower wall deformation rate and weak permeability. Besides, the presence of other nonperturbed physical parameters makes their solution more vulnerable as the perturbation solution strictly relies on the existence of very small parameters in equation.

In this study, we have tried to remove those physical constraints, which have been imposed in [13], only to insure the convergence of their perturbation solution. A probable invalidity of their solution for concentration profile has also been pointed out even for smaller values of wall deformation rate and the permeation Reynolds number.

We have used a well-known analytical method called Homotopy Analysis Method (HAM) [14-18] to guarantee the convergence of the analytical solution. To further ensure the validity of the solution, a numerical solution (fourthorder Runge-Kutta coupled with shooting method) has also been sought. The aforesaid numerical method has effectively been used in several studies [19-21]. We have compared the analytical and numerical results and have found an excellent agreement between them. Finally, the effects of physical parameters on temperature and concentration profiles are displayed and analyzed with the help of graphs accompanied by comprehensive discussions.

\section{Mathematical Analysis}

The laminar flow of a viscous incompressible fluid is taken into account, in a rectangular domain of infinite length, and having two deformable porous walls. The fluid may enter or exit, during the successive contractions/expansions, through the permeable walls. The leading end of the container is closed with a solid but flexible membrane, which deforms incorporation with the movement of the walls. A narrow gap assumption has been imposed; that is, the height $(2 h)$ of the channel is taken to be very small as compared to its width. It enables us to confine the whole problem in to a half domain and a planer cross section of the simulating domain is presented in Figure 1.

Both the walls are assumed to have different (from each other) permeability and to deform uniformly at a time dependent rate $\dot{h}(=d h / d t)$. The origin is taken at the center of channel as shown in Figure 1. The temperature and concentration both possess higher values at the lower wall. Following the aforementioned assumptions, the governing equations take the following form [12]:

$$
\begin{gathered}
\frac{\partial \bar{u}}{\partial \bar{x}}+\frac{\partial \bar{v}}{\partial \bar{y}}=0 \\
\frac{\partial \bar{u}}{\partial t}+\bar{u} \frac{\partial \bar{u}}{\partial \bar{x}}+\bar{v} \frac{\partial \bar{u}}{\partial \bar{y}}=-\frac{1}{\rho} \frac{\partial \bar{p}}{\partial \bar{x}}+v\left(\frac{\partial^{2} \bar{u}}{\partial \bar{x}^{2}}+\frac{\partial^{2} \bar{u}}{\partial \bar{y}^{2}}\right),
\end{gathered}
$$

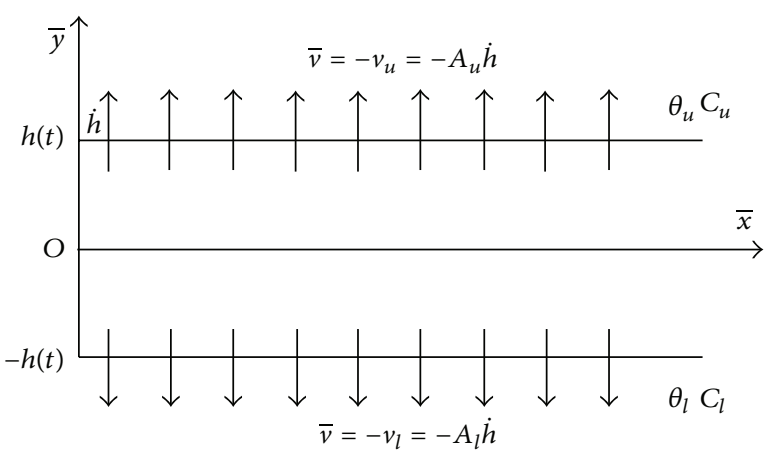

Figure 1: Schematic diagram of the problem.

$$
\begin{aligned}
\frac{\partial \bar{v}}{\partial t}+\bar{u} \frac{\partial \bar{v}}{\partial \bar{x}}+\bar{v} \frac{\partial \bar{v}}{\partial \bar{y}} & =-\frac{1}{\rho} \frac{\partial \bar{p}}{\partial \bar{y}}+v\left(\frac{\partial^{2} \bar{v}}{\partial \bar{x}^{2}}+\frac{\partial^{2} \bar{v}}{\partial \bar{y}^{2}}\right), \\
c_{p}\left(\frac{\partial \theta}{\partial t}+\bar{u} \frac{\partial \theta}{\partial \bar{x}}+\bar{v} \frac{\partial \theta}{\partial \bar{y}}\right) & =\frac{\kappa}{\rho} \frac{\partial^{2} \theta}{\partial \bar{y}^{2}} \\
\frac{\partial C}{\partial t}+\bar{u} \frac{\partial C}{\partial \bar{x}}+\bar{v} \frac{\partial C}{\partial \bar{y}} & =D \frac{\partial^{2} C}{\partial \bar{y}^{2}}-k_{1} C .
\end{aligned}
$$

In the above equations, the velocity components in $\bar{x}$ and $\bar{y}$ directions are denoted by $\bar{u}$ and $\bar{v}$, respectively. Moreover, $\bar{p}, \rho, c_{p}, v, \theta, \kappa, C, D$, and $t$ denote pressure, density, specific heat, kinematic viscosity, temperature, thermal conductivity, concentration, coefficient of mass diffusivity, and time, respectively. Further, $k_{1}$ represents the first-order chemical reaction rate taken to be positive for destructive reaction, negative for constructive reaction, and zero for no chemical reaction at all.

Suitable boundary conditions for the problem are as follows [12]:

$$
\begin{aligned}
& \bar{u}=0 ; \\
& \bar{v}=-v_{l}=-A_{l} \dot{h} ; \\
& \theta=\theta_{l} ; \\
& C=C_{l}, \quad \text { at } \bar{y}=-h(t), \\
& \bar{u}=0 ; \\
& \bar{v}=-v_{u}=-A_{u} \dot{h} ; \\
& \theta=\theta_{u} ; \\
& C=C_{u},
\end{aligned}
$$

where $A_{l}=v_{l} / \dot{h}$ and $A_{u}=v_{u} / \dot{h}$ denote the measure of permeability of lower and upper wall, respectively. Temperature and concentration are taken to be greater on the lower wall as compared to the upper wall; that is, $\theta_{l}>\theta_{u}$ and $C_{l}>C_{u}$. 
We can abridge the governing system of equations by eliminating the pressure terms from (2) and (3) and utilizing (1). Introducing vorticity $\bar{\omega}$, we get

$$
\frac{\partial \bar{\omega}}{\partial t}+u \frac{\partial \bar{\omega}}{\partial \bar{x}}+v \frac{\partial \bar{\omega}}{\partial \bar{y}}=v\left(\frac{\partial^{2} \bar{\omega}}{\partial \bar{x}^{2}}+\frac{\partial^{2} \bar{\omega}}{\partial \bar{y}^{2}}\right),
$$

where

$$
\bar{\omega}=\frac{\partial \bar{v}}{\partial \bar{x}}-\frac{\partial \bar{u}}{\partial \bar{y}} .
$$

Conservation of mass enables us to develop a similarity solution with respect to $\bar{x}$ as follows:

$$
\begin{aligned}
& y=\frac{\bar{y}}{h}, \\
& \bar{u}=v \bar{x} h^{-2} \bar{F}_{y}, \\
& \bar{v}=-v h^{-1} \bar{F}(y, t),
\end{aligned}
$$

where $\bar{F}_{y}$ represents $\partial \bar{F} / \partial y$. Using (9), we obtain

$$
\bar{\omega}=-v \bar{x} h^{-3} \bar{F}_{y y} .
$$

Using (10) in (7), we have

$$
\begin{aligned}
\bar{F}_{y y y y}+\alpha\left(3 \bar{F}_{y y}+y \bar{F}_{y y y}\right)-\bar{F}_{y} \bar{F}_{y y}+\bar{F}_{y y y} \\
-\frac{h^{2}}{v}\left(\bar{F}_{y y t}\right)=0,
\end{aligned}
$$

while $\alpha=h \dot{h} / v$ is the nondimensional wall expansion or contraction rate, taken to be positive for expansion.

We can set $\bar{F}_{y y t}=0$ by taking $\alpha$ to be a constant or a quasi constant in time [3].

The auxiliary conditions can also be transformed as

$$
\begin{gathered}
\bar{F}(-1, t)=R_{l} ; \\
\bar{F}_{y}(-1, t)=0, \\
\bar{F}_{y}(1, t)=0, \\
\bar{F}(1, t)=R,
\end{gathered}
$$

where $R_{l}=-v_{l} h / v$ and $R=v_{u} h / v$ are the permeation Reynolds numbers taken to be positive for injection.

Aforementioned formulation can be made nondimensional by introducing the following normalizing parameters:

$$
\begin{gathered}
u=\frac{\bar{u}}{\dot{h}}, \\
v=\frac{\bar{v}}{\dot{h}}, \\
x=\frac{\bar{x}}{h}, \\
F=\frac{\bar{F}}{R} .
\end{gathered}
$$

Consequently, we have

$$
F^{(i v)}+\alpha\left(y F^{\prime \prime \prime}+3 F^{\prime \prime}\right)+R\left(F F^{\prime \prime \prime}-F^{\prime} F^{\prime \prime}\right)=0
$$

with

$$
\begin{gathered}
F(-1)=A ; \\
F^{\prime}(-1)=0 ; \\
F^{\prime}(1)=0 ; \\
F(1)=1
\end{gathered}
$$

where $A=-v_{l} / v_{u}$. The primes in the above equations represent the differentiation with respect to $y$.

The equations describing the temperature and concentration are [12]

$$
\begin{gathered}
\theta=\theta_{l}+B\left(\frac{\bar{x}}{h}\right)^{n} G(y), \\
C=C_{l}+E\left(\frac{\bar{x}}{h}\right)^{s} H(y),
\end{gathered}
$$

where $n$ and $s$ are the power law indices of temperature and concentration, respectively. Besides, $B$ and $E$ are the fluid constants.

Substituting (16) in (4) and (5) and by using $G=(\theta-$ $\left.\theta_{l}\right) /\left(\theta_{u}-\theta_{l}\right)$ and $H=\left(C-C_{l}\right) /\left(C_{u}-C_{l}\right)$, we have dimensionless equations for the temperature and concentration distributions as follows:

$$
\begin{aligned}
& G^{\prime \prime}+\operatorname{Pr} \alpha\left(y G^{\prime}+n G\right)+R \operatorname{Pr}\left(F G^{\prime}-n F^{\prime} G\right)=0, \\
& H^{\prime \prime}+\operatorname{Sc} \alpha\left(y H^{\prime}+s H\right)+R \operatorname{Sc}\left(F H^{\prime}-s F^{\prime} H\right) \\
& \quad-\lambda \operatorname{Sc} H-k \operatorname{Sc}=0 .
\end{aligned}
$$

The boundary conditions become

$$
\begin{array}{r}
G(-1)=1 ; \\
G(1)=0 ; \\
H(-1)=1 ; \\
H(1)=0 .
\end{array}
$$

Pr in the above equation denotes Prandtl number, Sc is Schmidt number, $\lambda=k_{1} h^{2} / v$ is the chemical reaction parameter, and $k=k_{1} C_{l} h^{2} / v\left(C_{u}-C_{l}\right)$. It is also appropriate to mention that the similarity transforms given in (16) are only valid if $n=s=0$. We still retain the terms involving $n$ and $s$ in (17) to make them comparable with the work done by Reddy et al. [12]. However, the forthcoming analysis is done after correcting the values of these parameters. 


\section{Homotopy Solution}

Pursuing the technique suggested by Liao $[13,14]$, we can define the following initial guesses:

$$
\begin{aligned}
& F_{0}(y)=\frac{1}{4}\left(2+2 A+3(1-A) y+(A-1) y^{3}\right), \\
& G_{0}(y)=\frac{1-y}{2}, \\
& H_{0}(y)=\frac{1-y}{2} .
\end{aligned}
$$

These operators satisfy the following properties:

$$
\begin{aligned}
L_{F}\left(\xi_{1}+\xi_{2} y+\xi_{3} y^{2}+\xi_{4} y^{3}\right) & =0, \\
L_{G}\left(\xi_{5}+\xi_{6} y\right) & =0, \\
L_{H}\left(\xi_{7}+\xi_{8} y\right) & =0,
\end{aligned}
$$

where $\xi_{i}(i=1-8)$ are the arbitrary constants.

3.1. Zero-Order Deformation Problem. Representing $q \in$ $[0,1]$ as the embedding parameter, zero-order deformation problem can be constructed as

$$
\begin{aligned}
& (1-q) L_{F}\left[\widetilde{F}(y, q)-F_{0}(y)\right]=q h_{F} N_{F}[\widetilde{F}(y, q)], \\
& \widetilde{F}(-1, q)=A ; \\
& \widetilde{F}^{\prime}(-1, q)=0 ; \\
& \widetilde{F}(1, q)=1 ; \\
& \widetilde{F}^{\prime}(1, q)=0, \\
& (1-q) L_{G}\left[\widetilde{G}(y, q)-G_{0}(y)\right]=q h_{G} N_{G}[\widetilde{G}(y, q)], \\
& \widetilde{G}(-1, q)=1 ; \\
& \widetilde{G}(1, q)=0, \\
& (1-q) L_{H}\left[\widetilde{H}(y, q)-H_{0}(y)\right] \\
& \quad=q h_{H} N_{H}[\widetilde{H}(y, q)], \\
& \widetilde{H}(-1, q)=1 ; \\
& \widetilde{H}(1, q)=0,
\end{aligned}
$$

where $h_{F}, h_{G}$, and $h_{H}$ are the nonzero auxiliary parameter.
The nonlinear operators are

$$
\begin{aligned}
N_{F}[\widetilde{F}(y, q)]= & \frac{\partial^{4} \widetilde{F}}{\partial y^{4}}+\alpha\left(y \frac{\partial^{3} \widetilde{F}}{\partial y^{3}}+3 \frac{\partial^{2} \widetilde{F}}{\partial y^{2}}\right) \\
& +R\left(\widetilde{F} \frac{\partial^{3} \widetilde{F}}{\partial y^{3}}-\frac{\partial \widetilde{F}}{\partial y} \frac{\partial^{2} \widetilde{F}}{\partial y^{2}}\right), \\
N_{G}[\widetilde{G}(y, q)]= & \frac{\partial^{2} \widetilde{G}}{\partial y^{2}}+\operatorname{Pr} \alpha\left(y \frac{\partial \widetilde{G}}{\partial y}+n \widetilde{G}\right) \\
& +\operatorname{Pr} R\left(\widetilde{F} \frac{\partial \widetilde{G}}{\partial y}-n \widetilde{G} \frac{\partial \widetilde{F}}{\partial y}\right), \\
N_{G}[\widetilde{G}(y, q)]= & \frac{\partial^{2} \widetilde{H}}{\partial y^{2}}+\operatorname{Sc} \alpha\left(y \frac{\partial \widetilde{H}}{\partial y}+s \widetilde{H}\right) \\
& +\operatorname{Sc} R\left(\widetilde{F} \frac{\partial \widetilde{H}}{\partial y}-s \widetilde{H} \frac{\partial \widetilde{F}}{\partial y}\right) \\
& -\operatorname{Sc}\left(\lambda \widetilde{H}+K K_{1}\right) .
\end{aligned}
$$

3.2. $m$ th-Order Deformation Problem. The $m$ th-order problems satisfy

$$
\begin{aligned}
& L_{F}\left[F_{m}(y)-\chi_{m} F_{m-1}(y)\right]=h_{F} \mathfrak{R}_{m}^{F}(y), \\
& L_{G}\left[G_{m}(y)-\chi_{m} G_{m-1}(y)\right]=h_{G} \mathfrak{R}_{m}^{G}(y), \\
& L_{H}\left[H_{m}(y)-\chi_{m} H_{m-1}(y)\right]=h_{H} \mathfrak{R}_{m}^{H}(y), \\
& F_{m}(-1)=F_{m}^{\prime}(-1)=F_{m}(1)=F_{m}^{\prime}(1)=0, \\
& G_{m}(-1)=G_{m}(1)=0, \\
& H_{m}(-1)=H_{m}(1)=0,
\end{aligned}
$$

where

$$
\begin{aligned}
\mathfrak{R}_{m}^{F}(y)= & F_{m-1}^{(i v)}(y)+\alpha\left(y F_{m-1}^{\prime \prime \prime}+3 F_{m-1}^{\prime \prime}\right) \\
& +R\left(\sum_{k=0}^{m-1}\left(F_{m-1-k} F_{k}^{\prime \prime \prime}-F_{m-1-k}^{\prime} F_{k}^{\prime \prime}\right)\right), \\
\mathfrak{R}_{m}^{G}(y)= & G_{m-1}^{\prime \prime}(y)+\operatorname{Pr} \alpha\left(y G_{m-1}^{\prime}+n G_{m-1}\right) \\
& +\operatorname{Pr} R\left(\sum_{k=0}^{m-1}\left(F_{m-1-k} G_{k}^{\prime}-n F_{m-1-k}^{\prime} G_{k}\right)\right), \\
\mathfrak{R}_{m}^{H}(y)= & H_{m-1}^{\prime \prime}(y)+\operatorname{Sc} \alpha\left(y H_{m-1}^{\prime}+s H_{m-1}\right) \\
& -\lambda \operatorname{Sc} H_{m-1}-k \mathrm{Sc} \\
& +\operatorname{Sc} R\left(\sum_{k=0}^{m-1}\left(F_{m-1-k} H_{k}^{\prime}-s F_{m-1-k}^{\prime} H_{k}\right)\right) .
\end{aligned}
$$


Also,

$$
\chi= \begin{cases}0, & m \leq 1, \\ 1, & m>1 .\end{cases}
$$

For $q=0$ and $q=1$, we have

$$
\begin{aligned}
& \widetilde{F}(y, 0)=F_{0}(y), \\
& \widetilde{F}(y, 1)=F(y), \\
& \widetilde{G}(y, 0)=G_{0}(y), \\
& \widetilde{G}(y, 1)=G(y), \\
& \widetilde{H}(y, 0)=H_{0}(y), \\
& \widetilde{H}(y, 1)=H(y) .
\end{aligned}
$$

Using Taylor's series in terms of $q$, one can get

$$
\begin{aligned}
& \widetilde{F}(y, q)=F_{0}(y)+\sum_{m=1}^{\infty} F_{m}(y) q^{m}, \\
& F_{m}(y)=\left.\frac{1}{m !} \frac{\partial^{m} F(y, q)}{\partial y^{m}}\right|_{q=0}, \\
& \widetilde{G}(y, q)=G_{0}(y)+\sum_{m=1}^{\infty} G_{m}(y) q^{m}, \\
& \widetilde{H}(y, q)=H_{0}(y)+\sum_{m=1}^{\infty} H_{m}(y) q^{m},\left.\frac{1}{m !} \frac{\partial^{m} G(y, q)}{\partial y^{m}}\right|_{q=0}, \\
& f_{m}(y)=\left.\frac{1}{m !} \frac{\partial^{m} f(y, q)}{\partial y^{m}}\right|_{q=0} .
\end{aligned}
$$

Substituting $q=1$ in the above equations, we obtain

$$
\begin{aligned}
& F(y)=F_{0}(y)+\sum_{m=1}^{\infty} F_{m}(y), \\
& G(y)=G_{0}(y)+\sum_{m=1}^{\infty} G_{m}(y), \\
& H(y)=H_{0}(y)+\sum_{m=1}^{\infty} H_{m}(y),
\end{aligned}
$$

where $F_{m}(y), G_{m}(y)$, and $H_{m}(y)$ can be obtained by solving set of (24) using computer software, Mathematica. Substituting these back into (29) gives us the final solution.

3.3. Convergence of the Solution. The solution obtained in the preceding subsection contains the auxiliary parameters $h_{F}, h_{G}$, and $h_{H}$; these are very essential to control and adjust the convergence of the series solution. To determine the

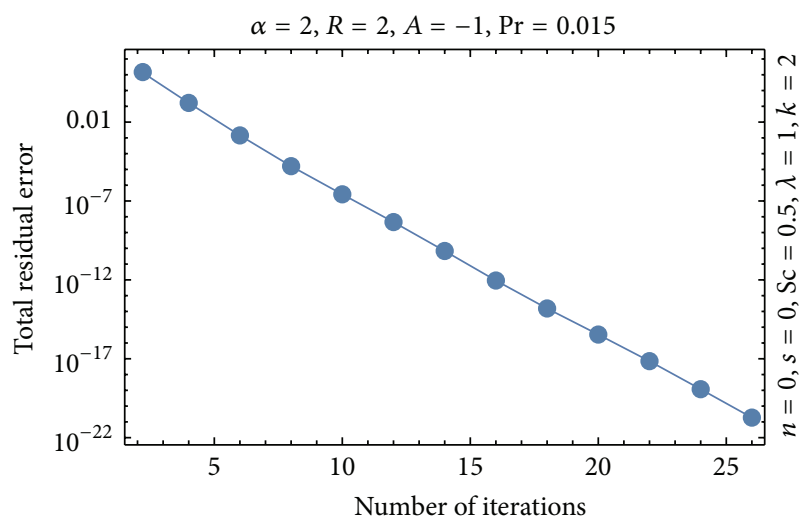

FIGURE 2: Total residual error with increasing order of approximations.

optimal values of these parameters so that the so-called total residual error is minimized, we have used a Mathematica package named BVPh 2.0 [14]. This is in accordance with the suggestions given by one of the respected anonymous reviewers. The resulting optimal values along with the total residual error, corresponding to different sets of physical parameters, are displayed in Table 1.

The convergence of the solution, for a specific set of physical parameters, is shown in Figure 2. A decline of total residual error with increasing the order of HAM iterations is evident. As the residual approaches zero quickly, it ensures the convergence of the solution.

The optimal values of the auxiliary parameters, which are far from -1, reveal that the solutions obtained by other methods such as homotopy perturbation method and Adomian's decomposition method are not suitable for this problem as they are suspected to diverge. The possible divergence is also an issue with the regular perturbation method. The solution presented in [12] is only valid in very limiting cases where the physical parameters are taken to be too small. Besides, they have only perturbed the wall deformation parameter $(\alpha)$ and permeation Reynolds number $R$. As there are other several physical parameters involved, the solution portrayed by [12] becomes more vulnerable. The solutions we obtained in this study are free from existence of small or large parameters, and also the convergence is assured by the use of optimal values of $h_{F}, h_{G}$, and $h_{H}$. Therefore, it seems right to proclaim that ours are the improved results.

The results are also compared with the numerical results obtained by using well-known fourth-order Runge-Kutta method. An excellent agreement between both the results has been documented in the form of Table 2.

\section{Results and Discussions}

The main objective of this section is to analyze the effects of emerging parameters on nondimensional temperature $(G(y))$ and concentration $(H(y))$ profiles. To serve this purpose, graphical simulation is presented in the figures to follow. The way in which the deformation parameter $\alpha$ and permeation 
TABLE 1: Optimal values of auxiliary parameters at 8th iteration.

\begin{tabular}{|c|c|c|c|c|c|c|c|c|c|c|c|c|}
\hline$\alpha$ & $R$ & $A$ & $\operatorname{Pr}$ & $\mathrm{Sc}$ & $\lambda$ & $k$ & $n$ & $s$ & $\hbar_{F}$ & $\hbar_{G}$ & $\hbar_{H}$ & Total error \\
\hline 2 & 1 & -1 & 0.015 & 0.5 & 1 & 2 & 0 & 0 & -1.023 & -0.802 & -0.840 & $2.065 \times 10^{-8}$ \\
\hline 1 & & & & & & & & & -0.932 & -1.150 & -0.726 & $7.387 \times 10^{-11}$ \\
\hline \multirow[t]{12}{*}{2} & 1.5 & & & & & & & & -0.910 & -1.239 & -0.751 & $3.301 \times 10^{-7}$ \\
\hline & 1.2 & & & & & & & & -0.932 & -0.792 & -0.843 & $1.042 \times 10^{-7}$ \\
\hline & 1 & -0.7 & & & & & & & -1.039 & -0.799 & -0.850 & $1.238 \times 10^{-8}$ \\
\hline & & -0.3 & & & & & & & -1.054 & -1.233 & -0.870 & $1.168 \times 10^{-8}$ \\
\hline & & -1 & 0.71 & & & & & & -1.023 & -1.058 & -0.840 & $2.063 \times 10^{-8}$ \\
\hline & & & 0.42 & & & & & & -1.023 & -0.793 & -0.841 & $2.065 \times 10^{-8}$ \\
\hline & & & 0.015 & 1 & & & & & -1.024 & -0.802 & -0.670 & $3.437 \times 10^{-8}$ \\
\hline & & & & 1.5 & & & & & -1.117 & -0.807 & -0.573 & $2.790 \times 10^{-6}$ \\
\hline & & & & 0.5 & 1.5 & & & & -1.023 & -0.802 & -0.800 & $1.994 \times 10^{-8}$ \\
\hline & & & & & 2 & & & & -1.023 & -0.802 & -0.739 & $2.032 \times 10^{-8}$ \\
\hline & & & & & 1 & 2.5 & & & -1.023 & -0.802 & -0.729 & $2.088 \times 10^{-8}$ \\
\hline & & & & & & 2.8 & & & -1.024 & -0.802 & -0.871 & $1.728 \times 10^{-8}$ \\
\hline
\end{tabular}

TABLE 2: The comparison between numerical and analytical solutions.

\begin{tabular}{|c|c|c|c|c|c|c|}
\hline \multirow{3}{*}{$\begin{array}{l}y \\
\downarrow\end{array}$} & \multicolumn{5}{|c|}{$\alpha=R=2, A=-1, \operatorname{Pr}=0.015, n=s=0, \mathrm{Sc}=0.5, \lambda=1$, and $k=2$} & \\
\hline & \multicolumn{2}{|c|}{$F(y)$} & \multicolumn{2}{|c|}{$G(y)$} & \multicolumn{2}{|c|}{$H(y)$} \\
\hline & HAM & Numerical & HAM & Numerical & HAM & Numerical \\
\hline-1 & -1.000000 & -1.000000 & 1.000000 & 1.000000 & 1.000000 & 1.000000 \\
\hline-0.9 & -0.991199 & -0.991199 & 0.950698 & 0.950698 & 0.896188 & 0.896188 \\
\hline-0.8 & -0.963137 & -0.963137 & 0.901190 & 0.901190 & 0.792370 & 0.792370 \\
\hline-0.7 & -0.913710 & -0.913710 & 0.851490 & 0.851490 & 0.689253 & 0.689253 \\
\hline-0.6 & -0.841553 & -0.841553 & 0.801619 & 0.801619 & 0.587744 & 0.587744 \\
\hline-0.5 & -0.746307 & -0.746307 & 0.751594 & 0.751594 & 0.488943 & 0.488943 \\
\hline-0.4 & -0.628838 & -0.628838 & 0.701439 & 0.701439 & 0.394126 & 0.394126 \\
\hline-0.3 & -0.491359 & -0.491359 & 0.651175 & 0.651175 & 0.304684 & 0.304684 \\
\hline-0.2 & -0.337421 & -0.337421 & 0.600830 & 0.600830 & 0.222053 & 0.222053 \\
\hline-0.1 & -0.171760 & -0.171760 & 0.550429 & 0.550429 & 0.147615 & 0.147615 \\
\hline 0.0 & 0.000000 & 0.000000 & 0.500000 & 0.500000 & 0.082594 & 0.082594 \\
\hline 0.1 & 0.171760 & 0.171760 & 0.449571 & 0.449571 & 0.027958 & 0.027958 \\
\hline 0.2 & 0.337421 & 0.337421 & 0.399170 & 0.399170 & -0.015655 & -0.015655 \\
\hline 0.3 & 0.491359 & 0.491359 & 0.348825 & 0.348825 & -0.047977 & -0.047977 \\
\hline 0.4 & 0.628838 & 0.628838 & 0.298561 & 0.298561 & -0.069111 & -0.069111 \\
\hline 0.5 & 0.746307 & 0.746307 & 0.248406 & 0.248406 & -0.079494 & -0.079494 \\
\hline 0.6 & 0.841553 & 0.841553 & 0.198381 & 0.198381 & -0.079835 & -0.079835 \\
\hline 0.7 & 0.913710 & 0.913710 & 0.148510 & 0.148510 & -0.071033 & -0.071033 \\
\hline 0.8 & 0.963137 & 0.963137 & 0.098810 & 0.098810 & -0.054097 & -0.054097 \\
\hline 0.9 & 0.991199 & 0.991199 & 0.049302 & 0.049302 & -0.030077 & -0.030077 \\
\hline 1.0 & 1.000000 & 1.000000 & $-3.139 \times 10^{-17}$ & $3.830 \times 10^{-11}$ & $-1.110 \times 10^{-16}$ & $9.961 \times 10^{-9}$ \\
\hline
\end{tabular}

Reynolds number $R$ affect the temperature and concentration profiles is depicted in Figures 3 and 4, respectively.

It is evident from Figure 3 that, for contracting walls $(\alpha<$ 0 ), the temperature drops in lower half of the channel with increasing contraction. Besides, the contraction combined with injection sends the heated fluid from lower wall to the upper quarter and as a result the temperature rises in upper quarter of the channel. For expanding walls $(\alpha>0)$, the phenomenon is reversed. As the walls expand, they create room; to fill it, the fluid near that region rushes in. The fluid near the lower wall bears higher temperature values due to the higher temperature of lower wall. On the other hand, the fluid near the upper wall becomes cooler due to the lower temperature of the upper wall. The same figure shows that the concentration profiles behave similarly to temperature profile for varying $\alpha$. In Figure $4, R<0$ corresponds to 


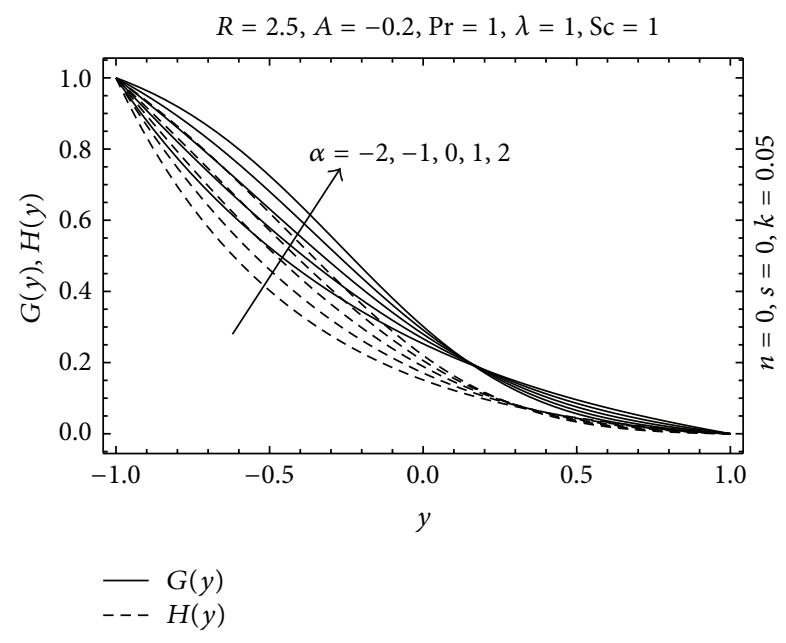

FIGURE 3: Effects of $\alpha$ on temperature and concentration profiles.

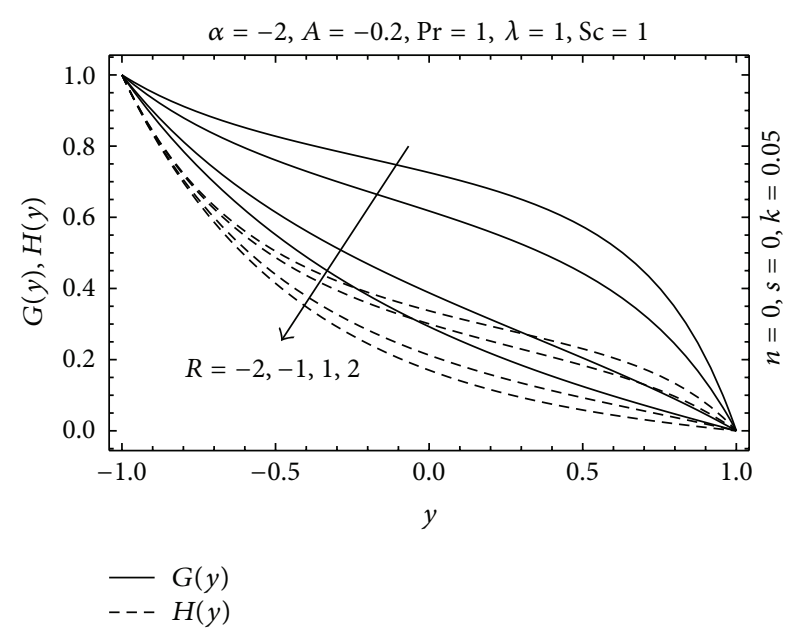

FIGURE 4: Effects of $R$ on temperature and concentration profiles.

the suction case, while $R>0$ represents the injection case. For contraction combined with suction, the temperature profile drops due to increased suction of the fluid by the walls. On the other hand, when contraction is combined with injection, the temperature distribution increases with increasing values of $R$. Again, the behavior of temperature and concentration profiles is similar.

For the case of expansion combined with injection, the influences of $A$ on temperature and concentration profiles are shown in Figure 5. As the value of $A$ increases from -1 to 0 , a decrease in both temperature and concentration distributions is observed. The expansion brings more fluid towards the walls. Moreover, with increasing $A$, the injection at upper wall plays a more dominating role as compared to the contribution from the lower wall. As a result, a drop in both the temperature and concentration profiles is expected. It is also pertinent to mention that the vanishing value of A means no injection at lower wall. An opposite behavior can be seen in Figure 6; the case of suction combined with contraction is displayed. As the suction at the lower wall

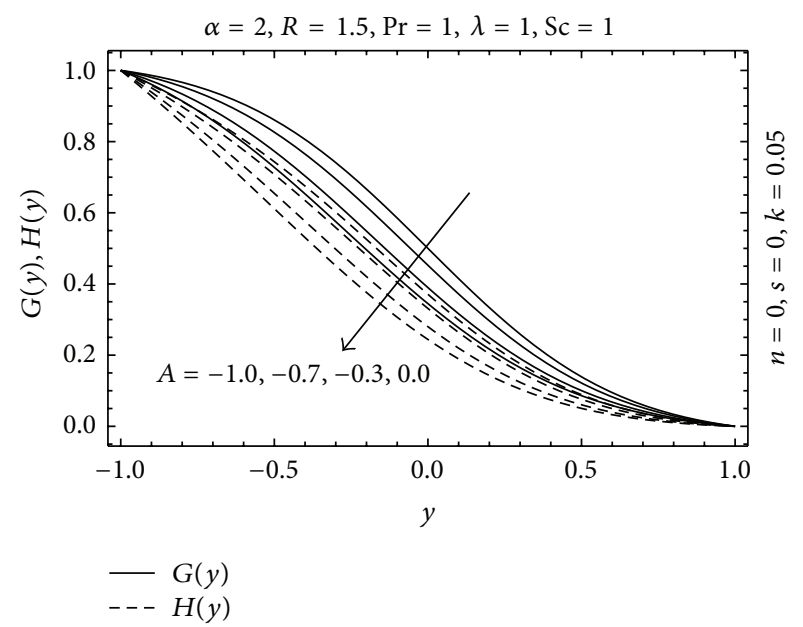

FIgURE 5: Effects of $A$ on temperature and concentration profiles (injection/expansion case).

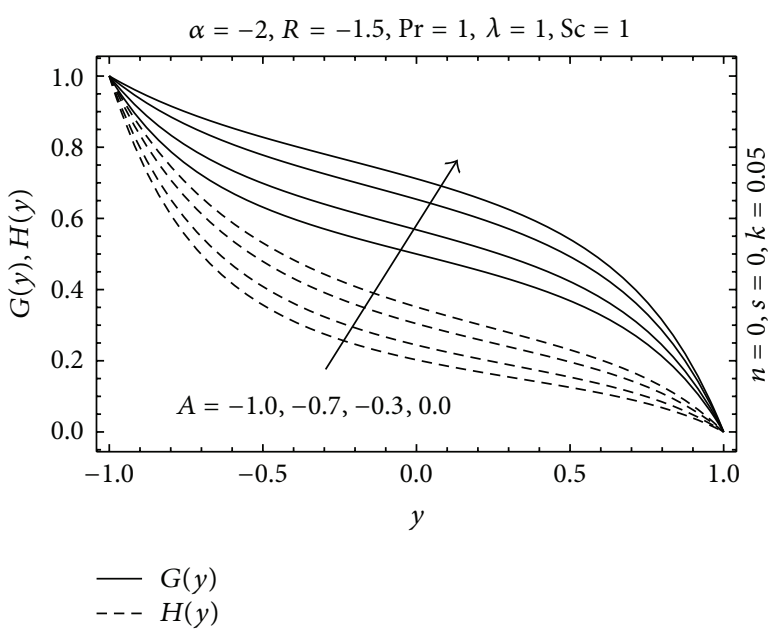

Figure 6: Effects of $A$ on temperature and concentration profiles (suction/contraction case).

decreases $(A \rightarrow 0)$ and also the contraction pushes the fluid inwards, the temperature as well as the concentration is likely to rise up.

The graphical results, unfolding the influence of Prandtl number on temperature profile, are presented in Figure 7. Different values of Prandtl number (i.e., $\operatorname{Pr}=0.015,0.71$, 4.2, 7.2, representing mercury, air, R-12 refrigerant, and sea water at $20^{\circ} \mathrm{C}$, resp.) are considered. For expansion combined with injection, an increase in $\mathrm{Pr}$ results in a temperature drop near the center and upper half of the channel. As Pr increases, the momentum diffusivity increases as compared to the thermal diffusivity; therefore, the temperature drops in the channel. However, the injection of heated fluid through lower wall increases the temperature in lower quarter of the channel. Figure 8 is dedicated to show the behavior of concentration profile under increasing Sc. The concentration is found to be a decreasing function of Sc. As Sc increases, 


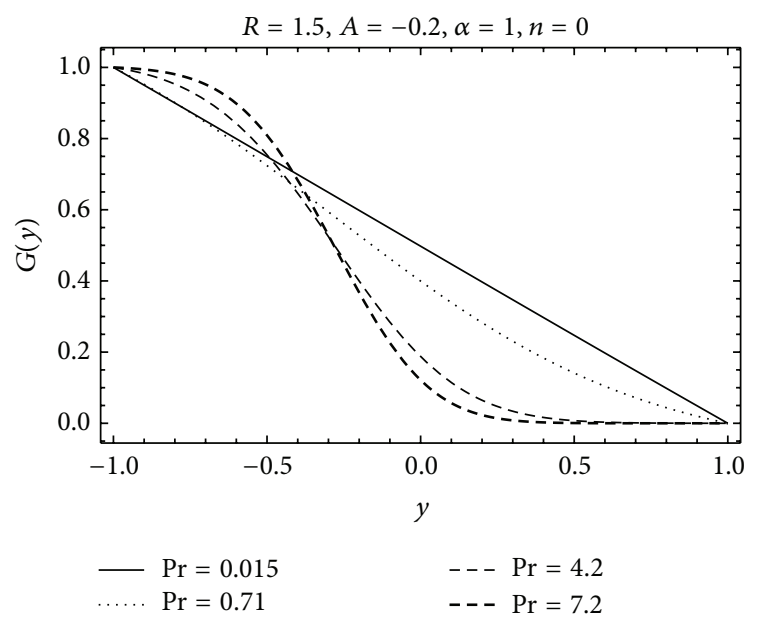

Figure 7: Effects of Pr on temperature profile (injection/expansion case).

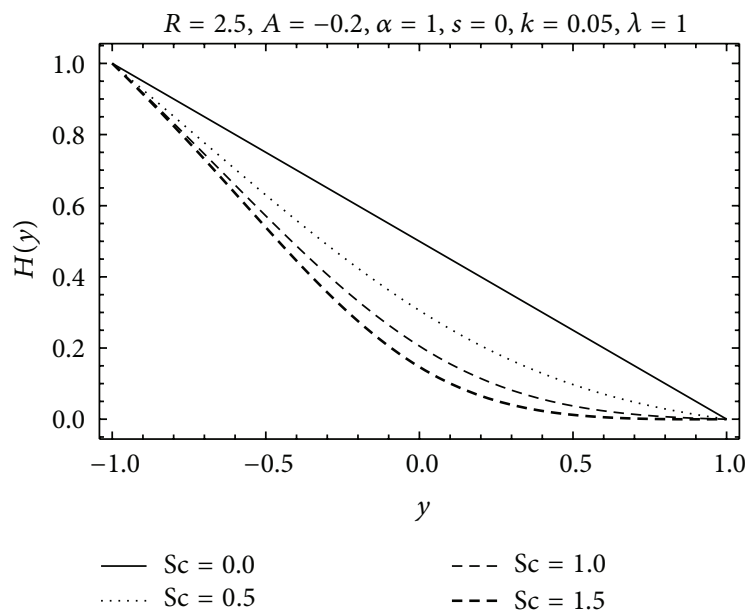

FIGURE 8: Effects of Sc on temperature profile (injection/expansion case).

mass diffusivity decreases; as a result, a concentration drop is exactly as expected.

Figures 9 and 10 show the behavior of concentration profile for constructive $(\lambda<0)$ and destructive $(\lambda>$ 0 ) chemical reactions, respectively. Suction combined with expansion is the case considered. For the generating chemical reaction, the concentration increases as absolute value of $\lambda$ increases. With increasing generation of radioactive solute, the concentration profile is expected to rise. On the other hand, this phenomenon is reversed for consuming chemical reaction. For that, the concentration is expected to drop with increasing rate of chemical reaction; this drop in concentration is clearly visible in Figure 10.

\section{Conclusions}

The simultaneous effects of heat transfer and chemical reaction are analyzed for the flow of a viscous fluid

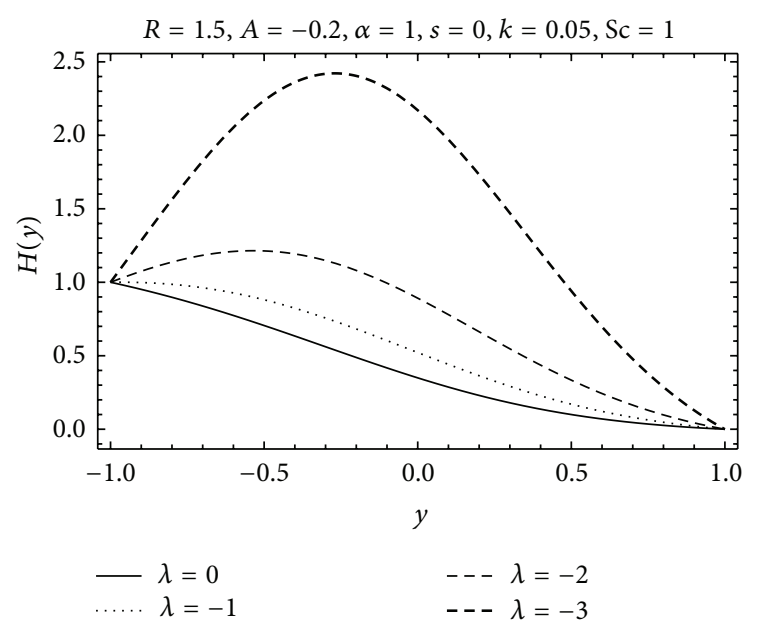

FIgURE 9: Effects of constructive $\lambda$ on concentration profile (suction/expansion case).

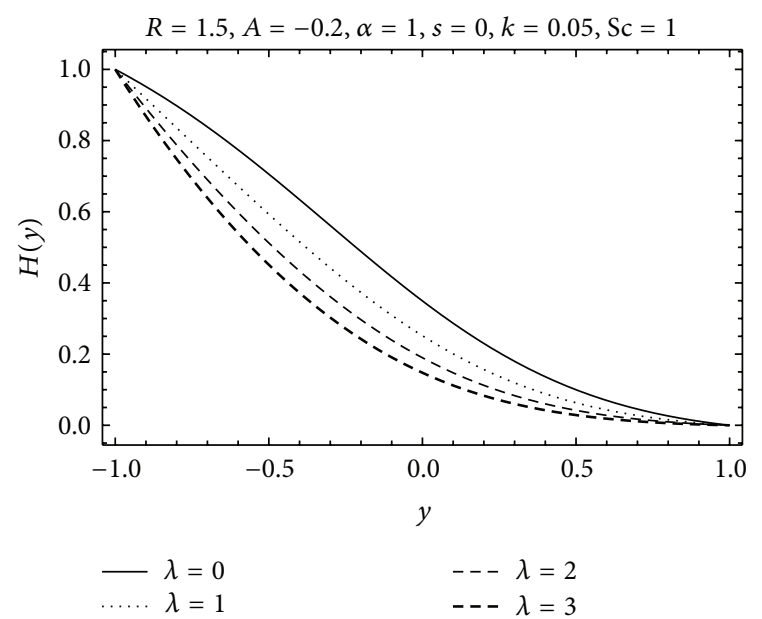

FIGURE 10: Effects of destructive $\lambda$ on concentration profile (suction/expansion case).

through a semi-infinite asymmetric channel with orthogonally deformable porous walls. A system of nonlinear ordinary differential equations that governs the flow is obtained by imposing the similarity transforms to the conservation laws. The resulting system of equations is solved both analytically and numerically, by using Homotopy Analysis Method (HAM) and fourth-order Runge-Kutta (RK-4) method, respectively. The convergence of analytical solution is ensured using the total squared residual analysis. By means of it, the optimal values of auxiliary parameters, involved in series solution, are obtained. A most recent study [12] is found to be a special case of ours because it is restricted only to very slow wall deformation and weak permeability cases. A possible divergence of their solution for concentration profile is also pointed out, even for smaller values of physical parameters. Our work is free from such overly imposed physical constraints and hence is more comprehensive. 


\section{Nomenclature}

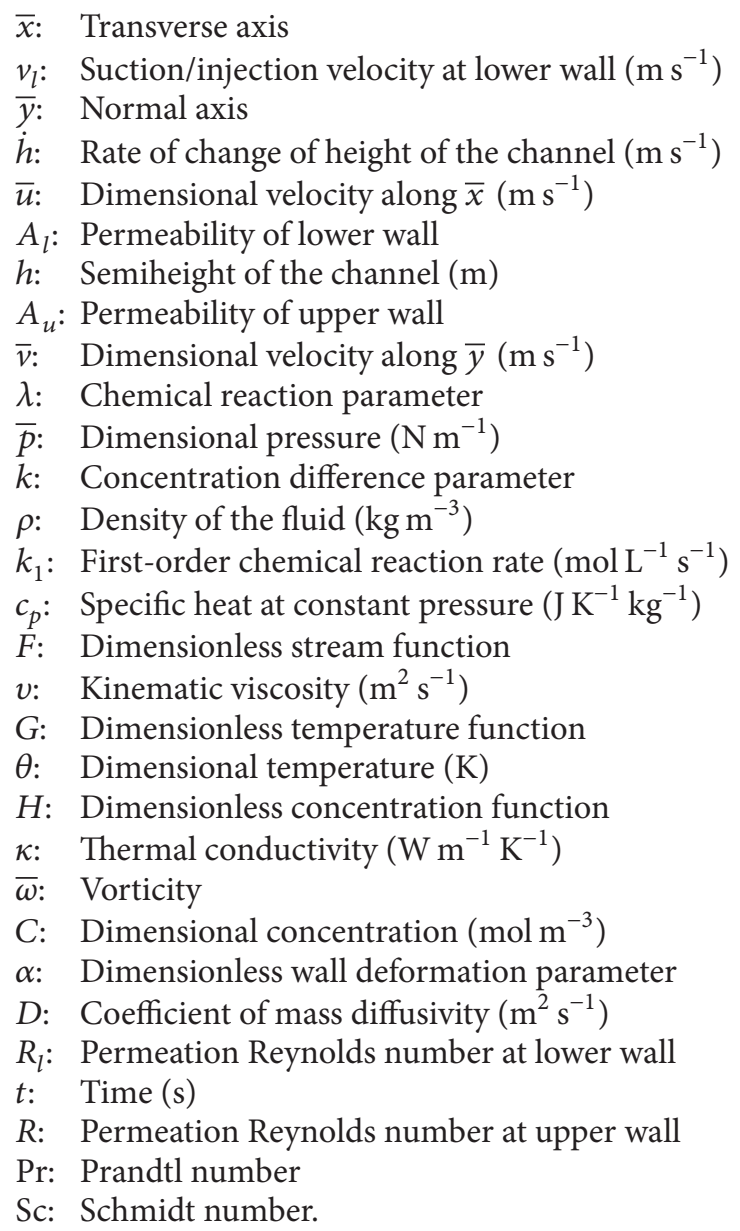

\section{Competing Interests}

The authors declare that they have no competing interests.

\section{Acknowledgments}

The author Maslina Darus is highly grateful to Universiti Kebangsaan Malaysia, 43600 Bangi, Selangor, for the necessary financial support via AP-2013-009.

\section{References}

[1] A. S. Berman, "Laminar flow in channels with porous walls," Journal of Applied Physics, vol. 24, pp. 1232-1235, 1953.

[2] J. Majdalani, C. Zhou, and C. A. Dawson, "Two-dimensional viscous flow between slowly expanding or contracting walls with weak permeability," Journal of Biomechanics, vol. 35, no. 10, pp. 1399-1403, 2002.

[3] C. Bridges and K. R. Rajagopal, "Pulsatile flow of a chemicallyreacting nonlinear fluid," Computers \& Mathematics with Applications, vol. 52, no. 6-7, pp. 1131-1144, 2006.

[4] S. Srinivas and R. Muthuraj, "Effects of chemical reaction and space porosity on MHD mixed convective flow in a vertical asymmetric channel with peristalsis," Mathematical and Computer Modelling, vol. 54, no. 5-6, pp. 1213-1227, 2011.
[5] N. Ahmed, S. T. Mohyud-Din, and S. M. Hassan, "Flow and heat transfer of nanofluid in an asymmetric channel with expanding and contracting walls suspended by carbon nanotubes: a numerical investigation," Aerospace Science and Technology, vol. 48, pp. 53-60, 2016.

[6] U. Khan, N. Ahmed, and S. T. Mohyud-Din, "Soret and dufour effects on flow in converging and diverging channels with chemical reaction," Aerospace Science and Technology, vol. 49, pp. 135-143, 2016.

[7] S. T. Mohyud-Din, Z. A. Zaidi, U. Khan, and N. Ahmed, "On heat and mass transfer analysis for the flow of a nanofluid between rotating parallel plates," Aerospace Science and Technology, vol. 46, pp. 514-522, 2015.

[8] S. Mohyud-Din, U. Khan, N. Ahmed, and S. M. Hassan, "Magnetohydrodynamic flow and heat transfer of nanofluids in stretchable convergent/divergent channels," Applied Sciences, vol. 5, no. 4, pp. 1639-1664, 2015.

[9] M. M. Rashidi, S. A. Mohimanian pour, and S. Abbasbandy, "Analytic approximate solutions for heat transfer of a micropolar fluid through a porous medium with radiation," Communications in Nonlinear Science and Numerical Simulation, vol. 16, no. 4, pp. 1874-1889, 2011.

[10] N. F. M. Noor, S. Abbasbandy, and I. Hashim, "Heat and mass transfer of thermophoretic MHD flow over an inclined radiate isothermal permeable surface in the presence of heat source/sink," International Journal of Heat and Mass Transfer, vol. 55, no. 7-8, pp. 2122-2128, 2012.

[11] U. Khan, N. Ahmed, and S. T. Mohyud-Din, "Heat transfer effects on carbon nanotubes suspended nanofluid flow in a channel with non-parallel walls under the effect of velocity slip boundary condition: a numerical study," Neural Computing and Applications, pp. 1-10, 2015.

[12] A. S. Reddy, S. Srinivas, and T. R. Ramamohan, "Analysis of heat and chemical reaction on an asymmetric laminar flow between slowly expanding or contracting walls," Heat TransferAsian Research, vol. 42, no. 5, pp. 422-443, 2013.

[13] S. J. Liao, Beyond Perturbation: Introduction to the Homotopy Analysis Method, CRC Press; Chapman \& Hall, Boca Raton, Fla, USA, 2003.

[14] S. Liao, Ed., Advances in the Homotopy Analysis Method, chapter 7, World Scientific Press, 2013.

[15] S. Abbasbandy and F. S. Zakaria, "Soliton solutions for the fifth-order KdV equation with the homotopy analysis method," Nonlinear Dynamics, vol. 51, no. 1-2, pp. 83-87, 2008.

[16] S. Abbasbandy, "The application of homotopy analysis method to solve a generalized Hirota-Satsuma coupled KdV equation," Physics Letters, Section A: General, Atomic and Solid State Physics, vol. 361, no. 6, pp. 478-483, 2007.

[17] S. Abbasbandy, "The application of homotopy analysis method to nonlinear equations arising in heat transfer," Physics Letters A, vol. 360, no. 1, pp. 109-113, 2006.

[18] T. Nawaz, A. Yildirim, and S. T. Mohyud-Din, "Analytical solutions Zakharov-Kuznetsov equations," Advanced Powder Technology, vol. 24, no. 1, pp. 252-256, 2013.

[19] N. Ahmed, U. Khan, S. I. Khan, Y. X. Jun, Z. A. Zaidi, and S. T. Mohyud-Din, "Magneto hydrodynamic (MHD) squeezing flow of a Casson fluid between parallel disks," International Journal of Physical Sciences, vol. 8, no. 36, pp. 1788-1799, 2013.

[20] U. Khan, N. Ahmed, and S. T. Mohyud-Din, "Thermo-diffusion, diffusion-thermo and chemical reaction effects on MHD flow of 
viscous fluid in divergent and convergent channels," Chemical Engineering Science, vol. 141, pp. 17-27, 2016.

[21] N. Ahmed, U. Khan, Z. A. Zaidi, S. U. Jan, A. Waheed, and S. T. Mohyud-Din, "MHD flow of an incompressible fluid through porous medium between dilating and squeezing permeable walls," Journal of Porous Media, vol. 17, no. 10, pp. 861-867, 2014. 


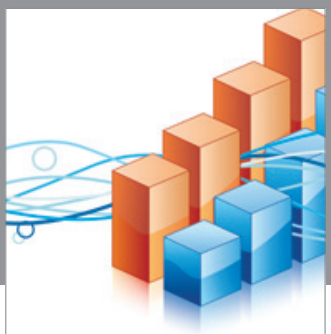

Advances in

Operations Research

vatem alat4

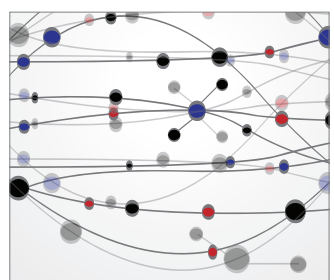

\section{The Scientific} World Journal
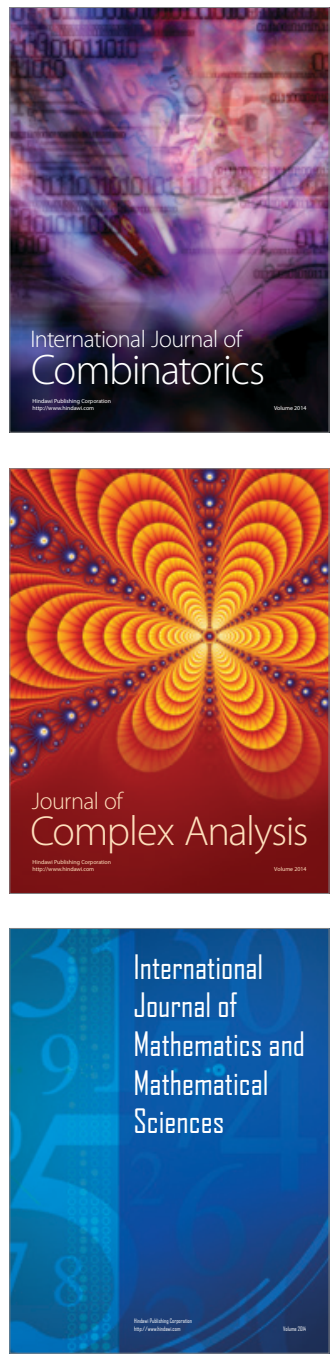
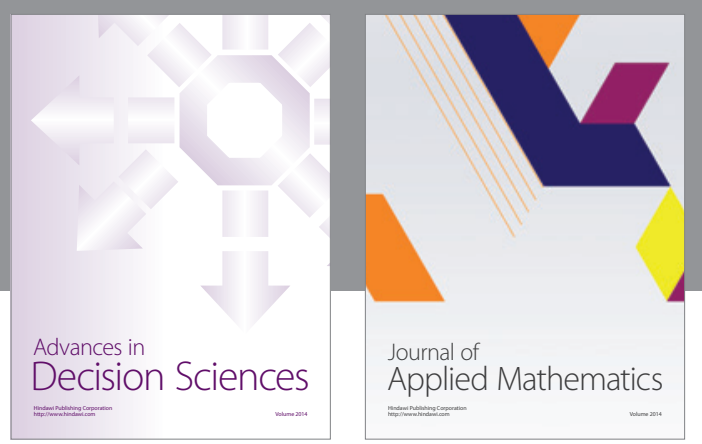

Algebra

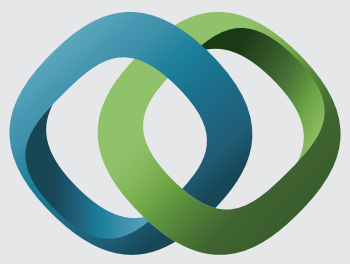

\section{Hindawi}

Submit your manuscripts at

http://www.hindawi.com
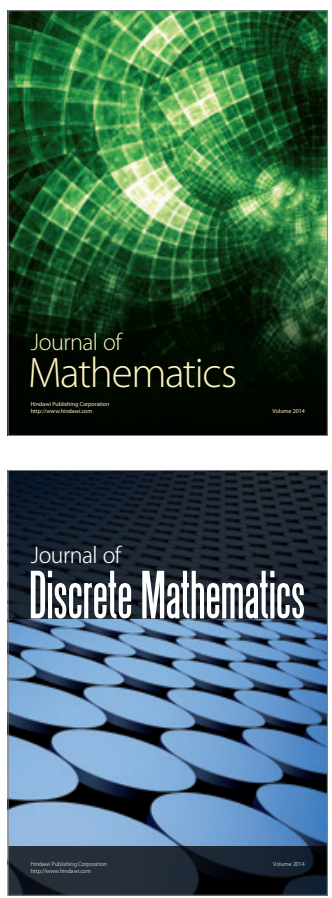

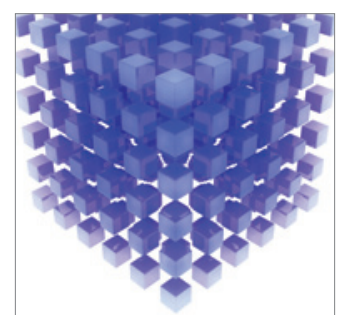

Mathematical Problems in Engineering
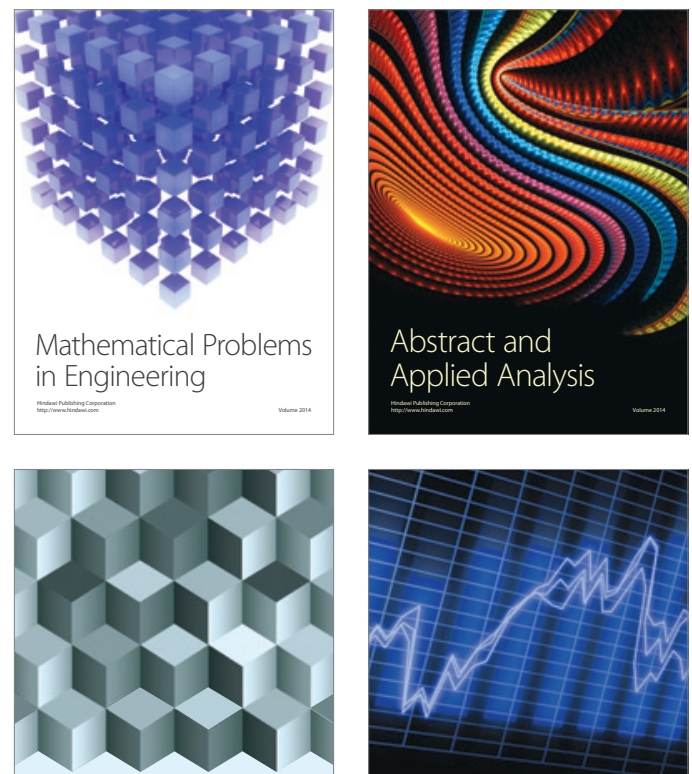

Journal of

Function Spaces

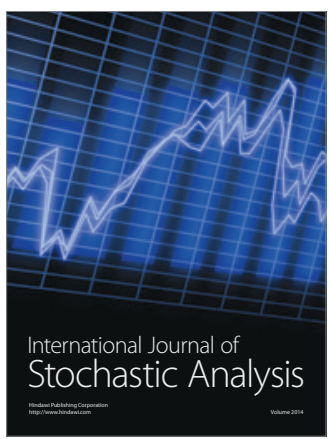

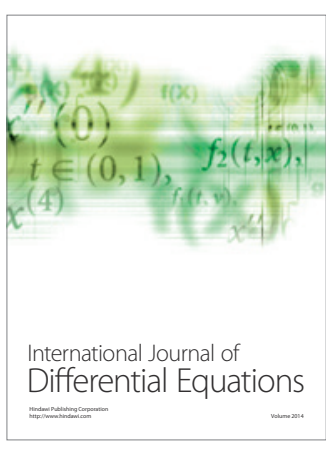
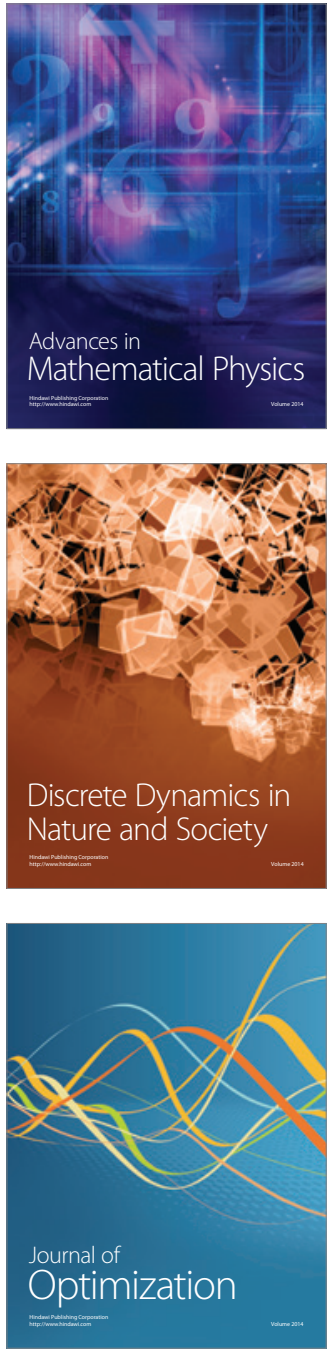